\title{
INFLUÊNCIA DO CANAL SÃO GONÇALO NO APORTE DE SEDIMENTOS PARA O ESTUÁRIO DA LAGUNA DOS PATOS - RS*
}

\author{
CARIOS HARIMANN** e PAULO FERNANDO CARRETA HARKOT**
}

\begin{abstract}
THE INFLUENCE OF SÃO GONCALO CHANNEL IN THE INFLOW OF THE SEDIMENTS TO PATOS LÀGGON ESTUARY-RS. During a fifteen hoars period environment and water quality observations have been realized in a fixe point at the mouth of Sao Goncalo Channel which flows into Patos Laggon. These observations have been done a few hours before and during a cold front that has affected the region and has determined important environment changes in the channel rate of flow because of wind action in a veloccity between 40 and $50 \mathrm{~km} / \mathrm{h}$. The changes mat have been verified in the inflow of the tide velocity and in the level of channel water this way reflecting themselves the erosion and transportation of the matter in suspension to the estuarine region, affecting the water quality. In this article, we show the results of this investigation which sticks to the normal standard sample because of roe difficulties imposed by the environment to mis kind of work.
\end{abstract}

Keywords: Sio Goncalo Cham», Patos Laggon, estuary, sediments, suspension, water quality.

\begin{abstract}
RESUMO Durante um período de 15 horas, realizaram-se observações ambientais e da qualidade da água em um ponto fixo na embocadura do Canal Sio Gonçalo, que deságua no estuário da Laguna dos Patos. As observações foram feitas horas antes e durante a ocorrência de uma frente fria que atingiu a região, determinando importantes mudanças ambientais no regime do canal pela ação de ventos com 40 e $50 \mathrm{~km} / \mathrm{h}$. As modificações verificadas na velocidade do fluxo e no nível da água do canal se refletiram na erosão e no transporte de sedimentos em suspensão para a região estuarina, afetando a qualidade da água. Neste artigo, são apresentados os resultados dessa investigação que foge aos padrões normais de amostragem pelas dificuldades impostas pelo meio a esse tipo de trabalho.
\end{abstract}

Palavras-chaves: Canal São Gonçalo, Laguna dos Patos, estuário, sedimentos, suspensão, qualidade da água.

INTRODUÇ̃̃O A desembocadura da Laguna dos Patos, devido às suas características próprias de mistura entre as águas doces (continentais) e salgadas (marinhas), determina, do ponto de vista antrópico, ofertas significativas de recursos biológicos renováveis, constituindo-se em ambiente de estuário.

Essas áreas possuem taxas de produção reguladas por intermédio de parâmetros abióticos, relacionados às características físico-químicas inerentes ao meio, que podem ser alteradas por eventos ambientais de caráter excepcional. Na área em estudo, as ações ambientais que mais afetam sua hidrologia estão relacionadas aos aspectos meteorológicos ligados à precipitação nas bacias de drenagem das Lagunas dos Patos e Mirim e às variações anemométricas locais (Hartmann et ai. 1986). As marés, apesar de quase inexpressivas (média anual de $47 \mathrm{~cm}$ ), têm sua influência aumentada quando em épocas de estiagem e quando auxiliadas por ventos do quadrante sul.

Este trabalho registra a brusca alteração ambiental presenciada na área, mais especificamente junto à desembocadura do Canal São Gonçalo (Fig. 1), causada por fortes ventos de sentido sudoeste com velocidades variando entre 40 e $50 \mathrm{~km} / \mathrm{h}$, por um período de doze horas, devido à ação de uma f rente fria que atingiu a região nas primeiras horas do dia 13 de julho de 1982. Tais ventos determinaram a ressuspensão de grandes quantidades de sedimentos das margens e do fundo do canal, em conseqüência do aumento da corrente, transportando-os em direção ao estuário da Laguna dos Patos, de tal forma que, em amostras de água coletadas na superfície, de três em três horas, num período de 15 horas, constatou-se que os $75 \mathrm{mg} / 1$ iniciais dos materiais em suspensão na região da desembocadura do Canal São Gonçalo passaram para $320 \mathrm{mg} / 1$ nas últimas cinco horas do início da amostragem. Tal fato se reveste de importância, porque, em relação à produtividade, a qualidade do meio hidríco decresce com o aumento da turbidez devido à

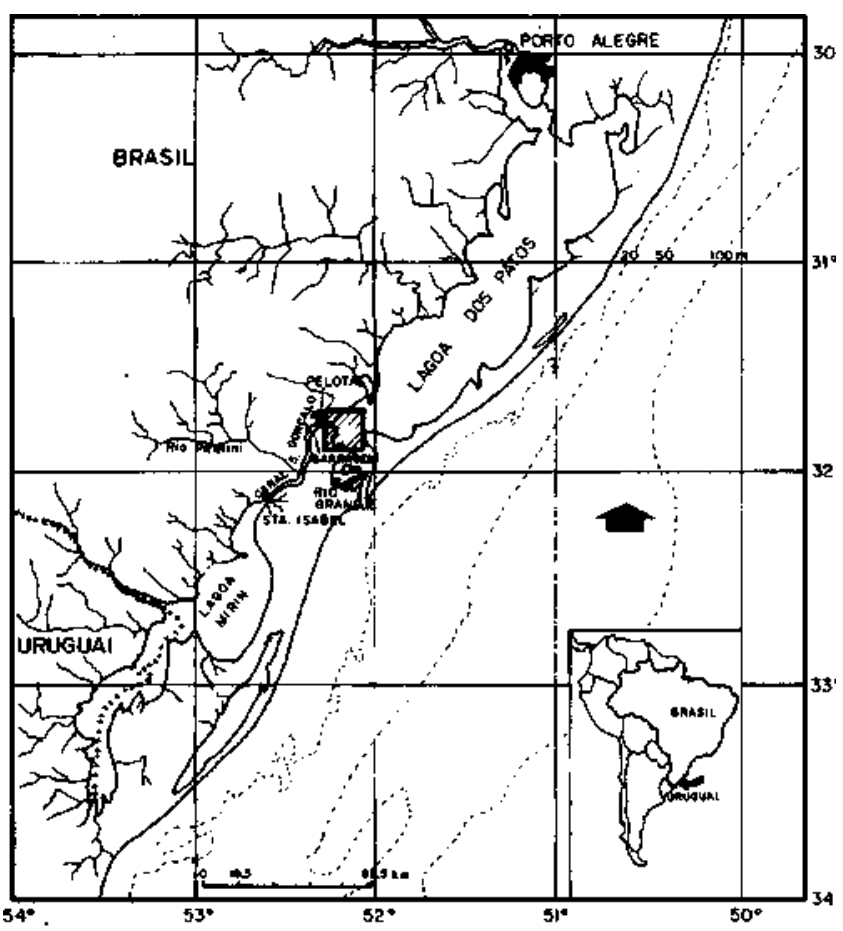

Figura I - Localizaçio da drea de estudo Figure 1 - Localization of the study are

diminuição da penetração de luz na coluna de água. A ressus-

\footnotetext{
* Financiado pela CIRM

** Departamento de Geociências, Fundação Universidade do Rio Grande, Caixa Postal 474, CEP 96200, Rio Grande, RS, Brasil
} 
pensão do material depositado provoca efeitos deletérios e uma série de impactos ambientais adversos ao meio ambiente aquático, de duas formas distintas:

1. diretamente, por propiciar a reentrada, no meio, dos mais diversos tipos de poluentes trapeados no sedimento, como agrotóxicos e insumos agrícolas, bem como metais pesados existentes em dejetos urbanos e industriais, além de outros tipos de poluentes existentes em menores concentrações; 2 . indiretamente, por aumentar a turbidez, diminuindo a penetração de luz na água, reduzindo a fotossíntese e, conseqüentemente, a produtividade (Clark 1977).

Por outro lado, as observações da qualidade da água são normalmente realizadas em situações normais, que permitem condições de trabalho in situ. As observações durante episódios de tempestade são evitadas pelas dificuldades impostas pelo meio, apesar de nestas condições, ocorrerem as maiores modificações na qualidade da água.

ÁREA DE ESTUDO A área de estudo localiza-se na região noroeste do estuário da Laguna dos Patos (Fig. 1), onde desemboca o Canal São Gonçalo. Este canal, com $70 \mathrm{~km}$ de extensão, intercomunica as Lagunas dos Patos e Mirim. O canal normalmente possui escoamento no sentido Laguna Mirim para a Laguna dos Patos. Esse fluxo, no entanto, pode inverter-se, o que geralmente ocorre em períodos de estiagem, quando esta for maior na bacia da Laguna Mirim.

MATERIAL E MÉTODOS Os dados utilizados neste trabalho foram adquiridos durante a amostragem na área, em 12 e 13 de julho de 1982, constituindo parte do Projeto MS Fase II (Hartmann 1985). Seis amostras de água foram coletadas na superfície (Fig. 2), utilizando-se um balde plástico. As amostras foram coletadas de três em três horas por um período de 15 horas. A água coletada foi acondicionada em garrafas plásticas de $250 \mathrm{ml}$, congeladas e posteriormente filtradas no laboratório sobre filtros de acetado de celulose de $0,45 \mathrm{xm}$ de poro e $45 \mathrm{~mm}$ de diâmetro. A concentração final nal do Material em Suspensão (MS), que constitui a fração inorgânica e orgânica do material particulado em suspensão, foi obtida por diferença de peso do filtro antes e após a filtração. Foi utilizado uma lupa binocular marca Zeiss para observação do MS retido no filtro, a qual permitiu comprovar a alta concentração de MS e a ocorrência de grãos minerais que são colocados em suspensão em situações de alta energia (Foto 1).

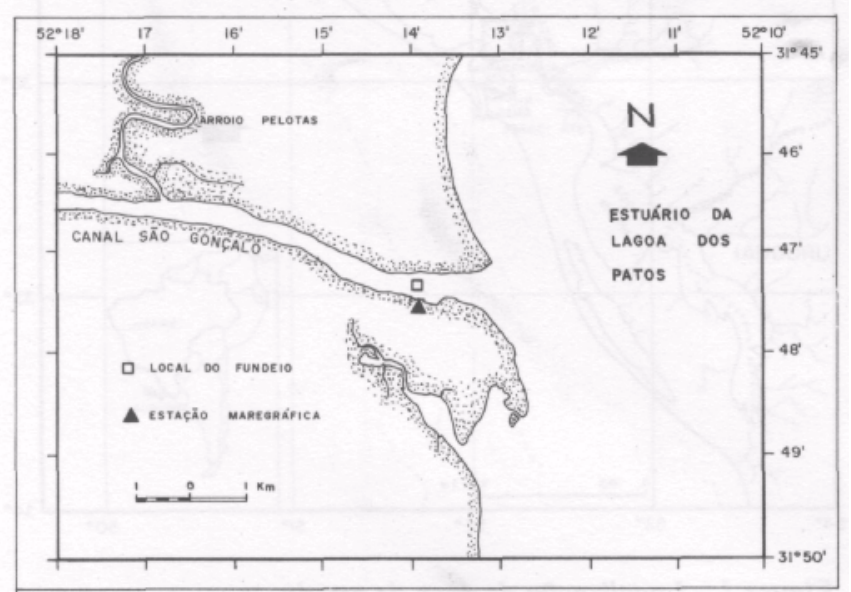

Figura 2 - Localização do sítio de amostragem Figure 2-Localization of the sample local

Registros da velocidade e da direção de correntes de superficie e a meia profundidade da coluna de água foram obtidos com a utilização de um correntômetro marca Hidrocean,

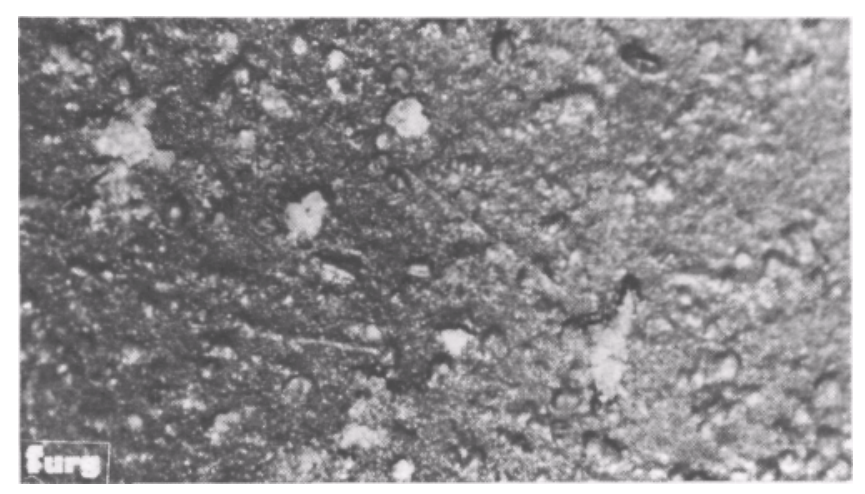

Foto 1 - Aspecto da concentração do material em suspensão retido no filtro. Amostra obtida às 8:30 h do dia 13/07/82. Observe-se a grande concentração de grãos minerais, de restos orgânicos e de material argiloso

Photo 1 - Concentration aspects of the suspended matter retained obtained at $8: 30 \mathrm{~h}$ of $13 / 07 / 1982$. We observe the great concentration of minerals grains, organic resíduo and clay material

modelo IRFE. A velocidade e direção do vento foram obtidas nos registros cedidos pelo Departamento Nacional de Águas e Energia Elétrica (DNAEE), a partir das estações de Rio Grande e agroclimatológica de Pelotas, pertencentes à Universidade Federal de Pelotas (UFPE1). Dados do nível de água (maré) na região da desembocadura do Canal São Gonçalo foram obtidos dos registros pertencentes ao Departamento de Portos, Rios e Canais (DEPREC), a partir de uma estação maregráfica existente na região (Fig. 2).

RESULTADOS E DISCUSSÃO A geologia e a geomorfologia da planície aluvial do Canal São Gonçalo foi revista por Vieira (1982), que estudou a sedimentologia do fundo do canal. As rochas fontes principais dos sedimentos que se acumularam na bacia costeira adjacente, que é parte da Planície Costeira do Rio Grande do Sul, são encontradas nas serras do susteste do Rio Grande do Sul, que compreendem o Escudo Cristalino Atlântico. Essas rochas fontes são constituídas por terrenos que integram o complexo granítico metamórfico de idade pré-cambriana. Quanto à distribuição granulométrica no fundo do canal, ocorre um aumento de granulometria com a profundidade, o que caracteriza um ambiente de canal, com maior intensidade de fluxo na sua parte central; os finos ocorrem nas áreas marginais mais rasas. Presume-se que, a partir da existência da barragem no Canal São Gonçalo, construída para evitar a penetração de água salgada e dessa forma evitar prejuízos às plantações de arroz irrigado, passaram a haver modificações na hidrodinâmica do canal com mudanças na distribuição granulométrica, principalmente em suas proximidades.

O escoamento da Laguna Mirim é avaliado diretamente na ligação do Canal São Gonçalo com a extremidade sudoeste da Laguna dos Patos (Fig. 1), que é sua desembocadura na região estuarina. A vazão desse canal depende do nível da água na Laguna dos Patos, das condições de fluxo do Rio Piratini (Fig. 1), do nível do canal na sua desembocadura e, mais recentemente, das condições impostas pela Barragem do Canal São 
Gonçalo; seu nível está relacionado, além dos fatores acima mencionados, com a ação dos ventos que, na maior parte das vezes, é o fator mais importante.

As variações periódicas que ocorrem sobre as condições hidrográficas da região são causadas pela evolução dos sistemas atmosféricos. Os sistemas que ocorrem com maior freqüência são os anticiclones migratórios (polares), que se deslocam seguindo uma trajetória de sudoeste para nordeste, com circulação no sentido anti-horário, induzindo a uma variação na direção do vento de sudoeste para nordeste (Paz 1985). Essa .variação do vento afeta diretamente o sistema de correntes da laguna e, sobretudo, o regime de enchentes e vazantes da região estuarina, como ocorreu em junho de 1984 (Hartmann et al 1986).

Por influir diretamente na zona estuarina da Laguna dos Patos, a análise do escoamento e do nível de descarga do canal é extremamente importante, devido à sua contribuição para a área de sólidos em suspensão, e de outros parâmetros como temperatura, $\mathrm{pH}$, Eh etc., que podem influir na qualidade da água. A esse respeito, diversas instituições ligadas à área vêm fazendo levantamentos com o objetivo de determinar os valores de vazão do canal. Um desses levantamentos realizados pela Comissão de Lagoa Mirim (CLM) (1970), órgão binacional (Brasil-Uruguai) para estudo da Laguna Mirim, registrou valores de vazão máxima de até $3.000 \mathrm{~m}^{3} / \mathrm{s}$, com médias em torno de $700 \mathrm{~m}^{3} / \mathrm{s}$ para uma seção média de $1.200 \mathrm{~m}^{2}$ e fluxos de vazante de $60 \mathrm{~cm} / \mathrm{s}$, em média. Outro levantamento, realizado na área do canal, e feito pela superintendência de Desenvolvimento do Extremo Sul (SUDE-SUL), determinou que a vazão média do canal num período de 30 anos (1939 a 1968) teve um valor de $566 \mathrm{~m}^{3} / \mathrm{s}$. Essa estimativa foi realizada com os níveis médios obtidos de dois locais, um localizado na extremidade oeste do Canal, em Santa Isabel, e outro, em Pelotas, pelo cálculo de seu desnível (Fig. 1).

Nas observações, as variações de fluxo do canal, relativas ao período considerado, são mostradas nos registros de nível (Fig. 3) e de direção e velocidade da corrente (Fig. 4), obtidas na área (Fig. 2). As variações que ocorrem na velocidade da corrente e no nível da água foram devidas às modificações atmosféricas resultantes da frente fria que atuou na região a partir do dia 12 de julho de 1982. O registro de nível (Fig. 3) apresenta com bastante evidência a variação da corrente, comprovada pelo registro simultâneo do correntógrafo (Fig. 4), no qual se constata uma resposta "causa-efeito" imediata.

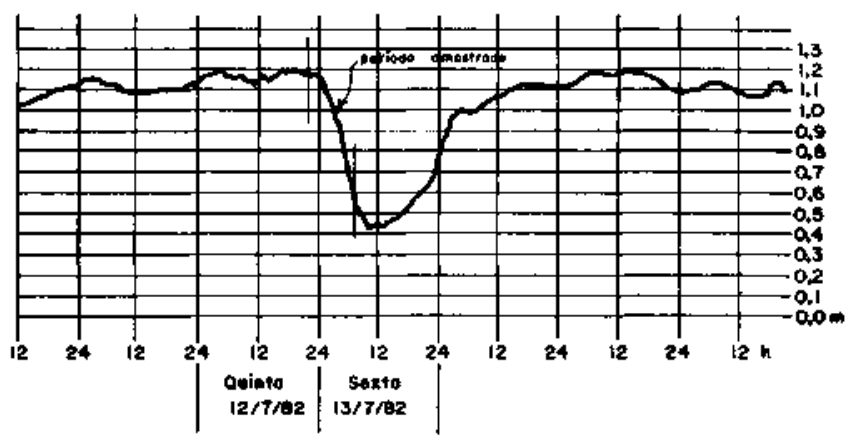

Figura 3-Maregrama - Barra do São Gonçalo Figure 3 - Maregram - SSo Gonçalo stady-bar

Para a situação analisada neste trabalho, a velocidade mínima da corrente foi da ordem de $25 \mathrm{~cm} / \mathrm{s}$ e a máxima de $85 \mathrm{~cm} / \mathrm{s}$ (Fig. 4), o que determina uma vazão, para uma seção média de $1.200 \mathrm{~m}^{3} / \mathrm{s}$, de $300 \mathrm{~m}^{3} / \mathrm{s}$ e de $1.020 \mathrm{~m}^{3} / \mathrm{s}$, respectivamente, e um valor médio de $660 \mathrm{~m}^{3} / \mathrm{s}$. O valor médio de $660 \mathrm{~m}^{3} / \mathrm{s}$ encontra-se bem próximo dos determinados pela

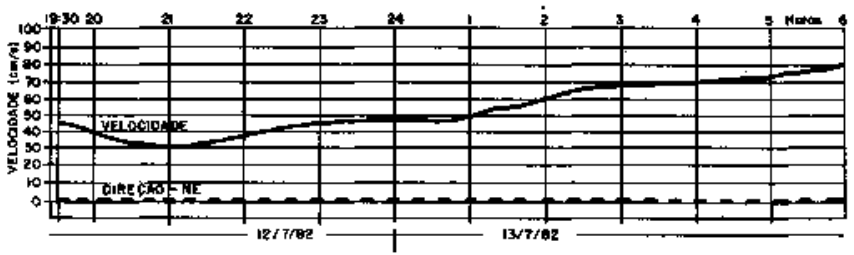
Figura 4-Registro de velocidade e direçao de corrente - Bar-
ra do Säo Gonçalo

Figuro 4 - Velocity date and current dhection - andy-bar Slo Goncalo Changel

CLM e SUDESUL, respectivamente, de $700 \mathrm{~m}^{3} / \mathrm{s}$.e $566 \mathrm{~m}^{3} / \mathrm{s}$. Considerando os totais de MS computados no período de amostragem (Fig. 7), mínimo de $75 \mathrm{mg} / 1$ e máximo de $320 \mathrm{mg} / 1$, é possível admitir que foram transportados para a região estuarina, para uma vazão média de $660 \mathrm{mVs}$, os totais de $495 \times 10^{-5}$ ton $/ \mathrm{s}$ ou 1,8 ton $/ \mathrm{h}$, e de $21 \times 10^{2}$ ton $/ \mathrm{s}$ ou $756 \mathrm{ton} / \mathrm{h}$, respectivamente para os valores mínimo e máximo de concentração de MS encontrados. Por outro lado, se for considerado o valor máximo de velocidade de corrente registrado no período, da ordem de $85 \mathrm{~cm} / \mathrm{s}$ e uma concentração de $320 \mathrm{mg} / 1$, constata-se que ingressou algo em torno de $33 \times 10^{-2}$ ton/s ou 1.175 ton $/ \mathrm{h}$ na região estuarina.

A influência da descarga do canal para a região estuarina pode ser observada no gráfico da figura 5. Verifica-se que, após um período de 14 horas (tempo observado), a distância percorrida pela massa d'agua foi de aproximadamente $36 \mathrm{~km}$,

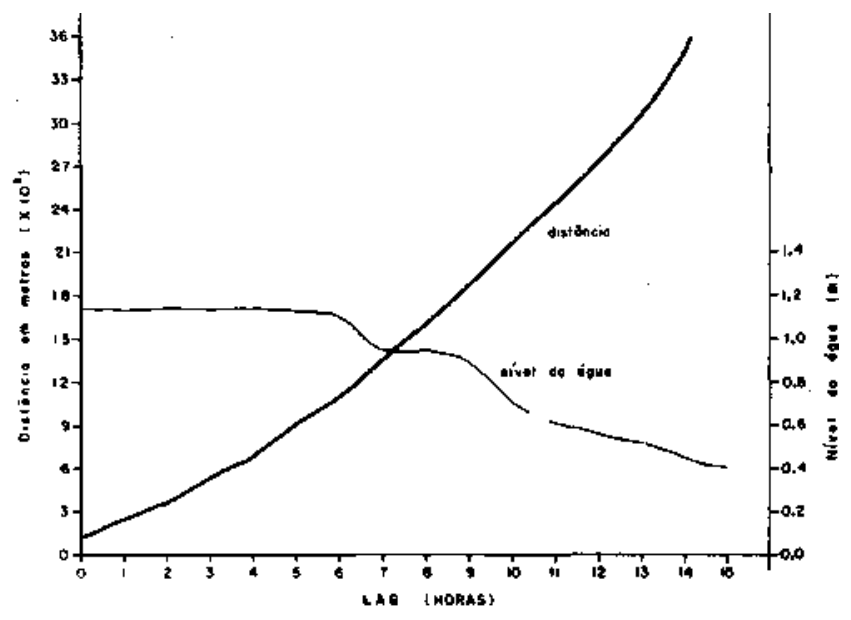

Figura 5 - Trajetória progressiva das correntes de superfície para a série de tempo (12 e 13/07/82), no Canal São Gonçalo, e nível da água

Figure S - Progressive boat-rote of the up flows to the time series (12 and 13/07/82) in the SSo Gonçalo Channel and water level

o que eqüivale à distância da barra do Canal São Gonçalo à barra da Laguna dos Patos, no oceano. Nesse mesmo gráfico (Fig. 5), é registrado que, após seis horas de observação, ocorre uma diminuição brusca do nível de água no canal, causada pelo aumento gradativo da velocidade da corrente e determinada pela força constante do vento na mesma direção do início das medidas, quando a velocidade era de $85 \mathrm{~cm} / \mathrm{s}$ (Fig. 4). Verifica-se, então, que o nível da água diminui para 
$56 \mathrm{~cm}$ (Fig. 5), o que representa uma diferença de $44 \mathrm{~cm}$ desde o início das observações.

Análises de correlação cruzada entre as velocidades da corrente e o nível da água com o time lag para séries de tempo permitem mostrar qual a influência da velocidade no nível em função do tempo, como mostrado por Hartmann (1988) na região do Canal de Rio Grande. Esse aumento gradativo do fluxo da corrente determina a diminuição do nível da água após um certo intervalo de tempo, este causado pela força da corrente na mesma direção.

No caso deste trabalho, apesar do número restrito de dados disponíveis, verifica-se correlação negativa em toda a série (Fig. 6), diminuindo de "lag O" $(\mathrm{R}=-0,98)$ para "lag 12" $(\mathrm{R}=-0,61)$, o que evidencia a influência da velocidade da corrente na diminuição do nível de água no canal, à medida que ocorre o aumento gradativo da velocidade da corrente, representando aumento de erosão e de transporte de sedimentos para a região estuarina da Laguna dos Patos (Fig. 6).

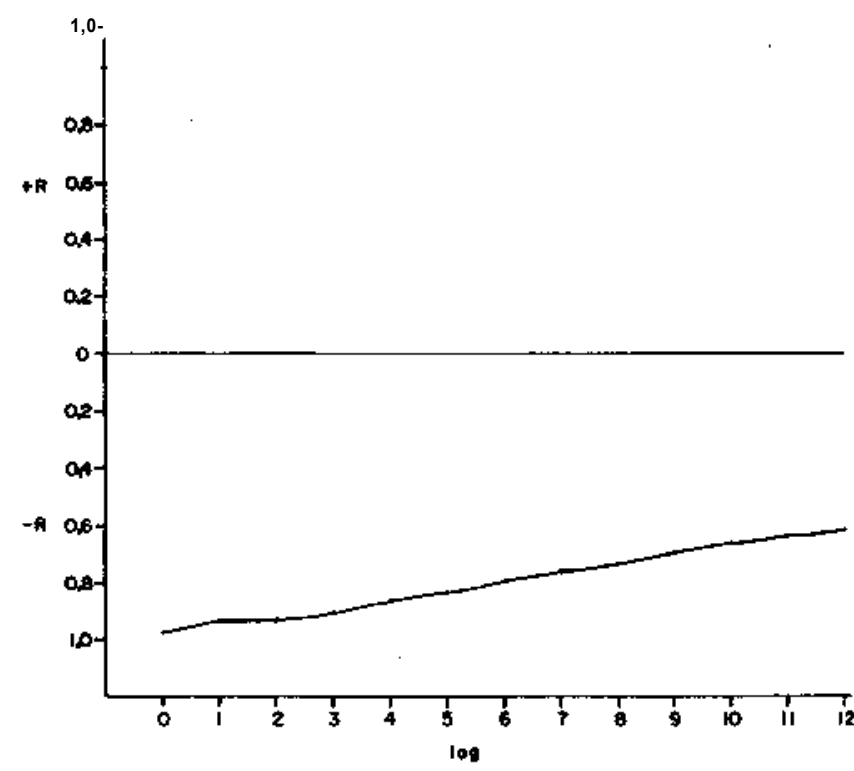

Figura 6 - Correlação em Time lag entre as velocidades de corrente de superficie, com o nível da água para a série de tempo (12 e 13/07/82)

Figure 6 - Correlaction with time lag between the upflow velocity the water level for the time serie (12 and 13/07/82)

O gráfico da figura 7 mostra que o aumento na concentração média de $75 \mathrm{mg} / 1$ para $320 \mathrm{mg} / 1$ corresponde a um acréscimo de $245 \mathrm{mg} / 1$ para um período de aproximadamente

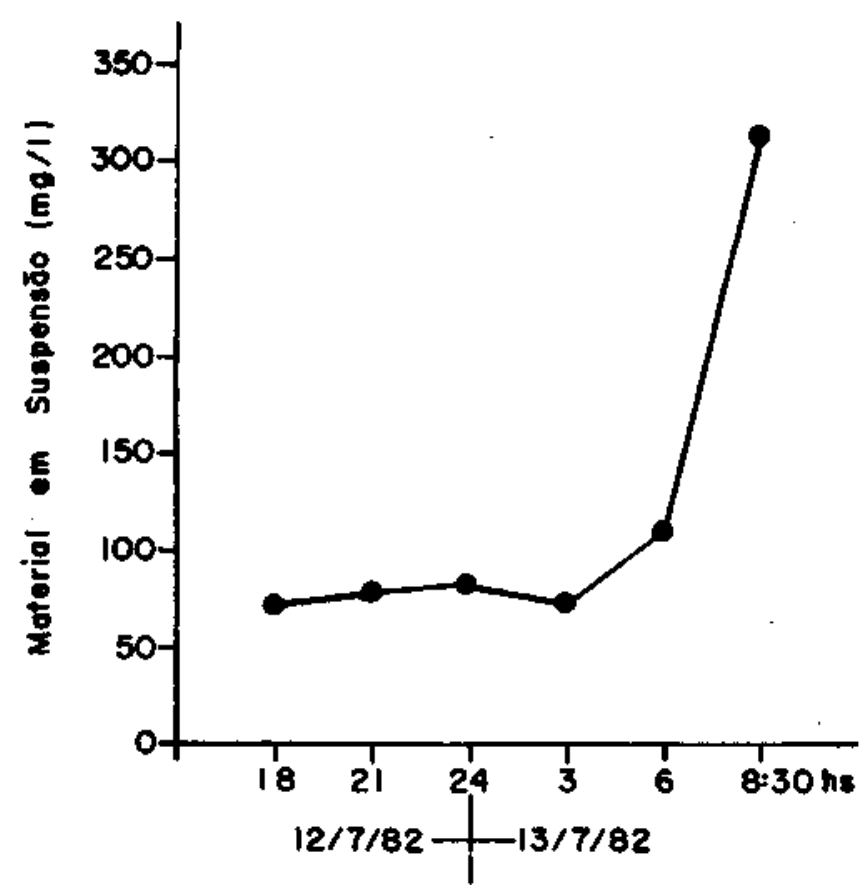

Figura 7 - Variação da concentração do Material em Suspensão em superfície na desembocadura do Canal São Gonçalo Figure 7 - Variation the concentration of the suspended matter in the mourn of Sã 0 Gonçalo Channel

cinco horas, demonstrando o trabalhamento e o transporte de sedimentos, no momento. Essa concentração de MS pode ser verificada na foto 1 , resultado de fotomicrografia do filtro em que se observa a ocorrência de grãos minerais em grande quantidade, além de material fino.

Finalmente, lembramos que os aspectos abordados neste trabalho mostram com evidência a influência dos fatores meteorológicos na hidrologia da região, principalmente quando tais fatores ocorrem de uma forma atípica e brusca, resultando, no caso, em rápidas variações no meio hídrico. Conclui-se, portanto, que são estas situações que mais promovem modificações no meio e que, apesar de sua importância para os aspectos mencionados, são de difícil quantificação.

Agradecimentos Os autores agradecem à tripulação da lancha Larus da Fundação Universidade do Rio Grande (FURG) e à Comissão Interministerial para os Recursos do Mar (CIRM).

\section{REFERÊNCIAS BIBLIOGRÁFICAS}

CLARK, J. E. 1977. Coastal Ecosystem Management (A technical manual for the conservation of coastal zone resources). New York, Wiley and Sons. 988p.

HARTMANN, C. 1985. Estudo do material em suspensão e dissolvido no estuário da Laguna dos Patos, RS. Fase tt. maio 1982 a dez.

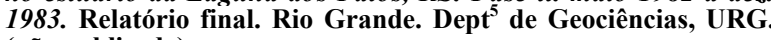
(não publicado).

HARTMANN, C.; SANO, E.E.; PAZ, R.S.; MOLLER Jr., O.0.1986. Avaliação de um período de cheia (junho 1984) na região sul da "Laguna dos Patos, através de dados de sensoriamento remoto, meteorológicos e oceanográficos. In: SIMP. BRAS. DE SENS REM., 10, Gramado, 1986. Anais... Gramado, SBSR. p.685-694.

HARTMANN, C. 1988 Utilização de dados dignais do mapeador temático para a obtenção dos padrões de distribuição do material em suspensão na desembocadura da Laguna dos Patos, RS. São José dos Campos. 190p. (Dissertação de Mestrado. INPE).

MINISTERIO DO INTERIOR. SEĈ̃̃O BRASILEIRA DA COMISSÃO DA LAGOA MIRIM. 1970. Barragem do Canal São Gonçalo; estudos preliminares de viabilidade. Generalidades e estudos básicos, v.2. (Relatório).
PAZ, R. da S. 1984. Aspectos meteorológicos da Lagoa dos Patos, RS. In: SEMIN. PESQ. LAGOA DOS PATOS, I, Porto Alegre, ES, 1984. (não publicado).

SUPERINTENDENCIA DE DESENVOLVIMENTO DO EXTREMO SUL. 1970. Dados de vazão do Canal São Gonçalo no período de 1939-1968. Porto Alegre, SUDESUL. (Rei. Técnico).

VIEIRA, H. 1982. Aspectos sedimentalógicos do Canal São Gonçalo. Porto Alegre. 132p. (Dissertação de Mestrado, UFRGS). 